\title{
SLIP Running with an Articulated Robotic Leg
}

\section{Conference Paper}

Author(s):

Hutter, Marco (D); Remy, C.David; Höpflinger, Mark A.; Siegwart, Roland

Publication date:

2010

Permanent link:

https://doi.org/10.3929/ethz-a-010184803

Rights / license:

In Copyright - Non-Commercial Use Permitted

Originally published in:

Proceedings of the International Conference on Intelligent Robots and Systems, https://doi.org/10.1109/IROS.2010.5651461 


\title{
SLIP Running with an Articulated Robotic Leg
}

\author{
Marco Hutter, C. David Remy, Mark A. Höpflinger, and Roland Siegwart, Fellow, IEEE
}

\begin{abstract}
SLIP models are generally known as one of the best and simplest abstractions describing the spring-like leg behavior found in human and animal running, and have thus been subject to exhaustive investigation. To exploit these findings in real robots, we utilize an operational space controller that projects the behavior of the SLIP model onto the dynamics of an actual segmented robotic leg. Additionally, we introduce a method to compensate for the energetic losses at the impact collisions, which are not accounted for in the simplified SLIP assumptions. This allows the direct application of existing dead-beat control strategies to arbitrary robotic legs, for which we can show that the collision and compensation effects in the actual leg enlarge the regions of stable running and reduce the minimally required locomotion speed. The necessary joint torque profiles can be generated in large part passively, for example by using high compliance series elastic actuators.
\end{abstract}

\section{INTRODUCTION}

$\mathrm{B}$ IOMECHANICAL studies suggest that the dynamics of the center of mass in running gaits (which include bounding, trotting, or galloping) can be described by the model of a spring loaded inverted pendulum (SLIP) [1, 2], which combines a prismatic mass-less elastic leg with a point mass at the Center of Gravity (CoG) (Fig. 1a). Experiments have shown that this model appropriately represents the $\mathrm{CoG}$ motion of a large variety of animals and humans, and also produces ground reaction forces that closely match experimental recordings $[3,4]$.

The discovery of using elastic legs for running gaits has quickly found its way into robotics. Springs and pneumatic pistons were used instead of muscles and tendons $[5,6]$ to periodically store energy during the ground contact phase, recover it for take-off, and to protect the actuators and gears from hard impacts at landing. Prismatic robotic legs developed along the lines of Marc Raibert's seminal hoppers [7] resemble SLIP models not only in terms of their mechanical design, but also from a dynamics point of view [8-10].

In biomechanical research, theoretical studies focused on two major aspects: Firstly, it was found that for certain parameter choices, the SLIP model is dynamically stable for single leg hopping [8-11] and walking [12]. This means, that when the state of the model is disturbed, the robot will

Manuscript received March 9, 2010. This work was supported in part by the Swiss National Science Foundation (SNF) (project 200021_119965/1).

Marco Hutter, C.David Remy, Mark A. Höpflinger, and Roland Siegwart are with the Autonomous Systems Lab, Institute of Robotics and Intelligent Systems, Swiss Federal Institute of Technology (ETHZ), Zurich, Switzerland (+41 $44 \quad 632 \quad 74 \quad$ 17; fax: +41 $44 \quad 632 \quad 11 \quad 81$; e-mail: mahutter@ethz.ch).
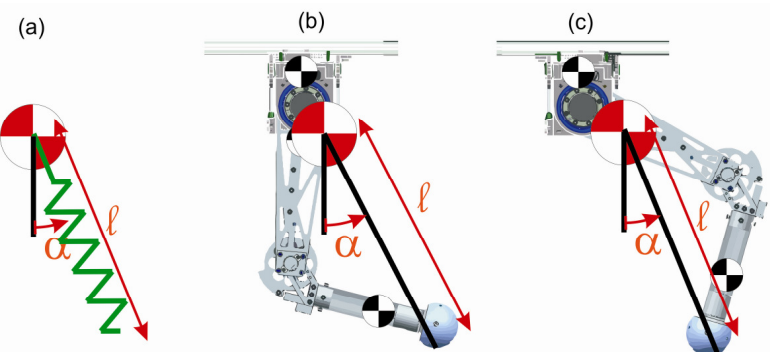

Fig. 1. In this study, we impose the dynamics of the SLIP model (shown in a) on a model of an articulated leg in backward (b) and forward (c) configuration. A low level operational space controller takes care of the dynamic coupling due to inertial effects, and a virtual spring pre-compression compensates for the impact losses at touchdown.

asymptotically return to its periodic steady state motion. The same effect has also been reported for 3D models [13]. In addition to the passive dynamic self stabilization, various control strategies have been proposed in order to increase the rate at which disturbances are rejected and to enlarge the basin of attraction of the periodic motion. Dead-beat controllers allow disturbance rejection within a single hopping period by appropriate adjustment of the angle of attack or stiffness of the leg spring [14]. This means that a constant velocity and apex height can be maintained continuously, even in extremely unstructured terrain [15].

In contrast to the prismatic elasticity that is assumed in the SLIP model, all biological and most robotic legs have a segmented structure with elasticities that act torsionally in their joints. Studies using simplified models with mass-less leg segments showed that stable periodic running with such segmented legs is possible when the joint stiffness is properly adjusted [16]. Additionally, the nonlinear forcetorque relation that results from the leg segmentation can lead to a substantial increase of stable regions of operation [17]. Yet, in a real robotic leg, as well as in biological limbs, the mass and inertia of the individual segments dynamically couple the motion of the segments, and lead to impactcollisions at touchdown, which are associated with energetic losses. These are two effects that have a large influence on the CoG motion and should hence not be neglected.

In this paper we strive to close the bridge between real robotic legs and the theoretical foundation provided by the SLIP model. To this end, we use a low level operational space controller [18] that forces the CoG motion of an arbitrary segmented leg to behave exactly like its equivalent SLIP model, and introduce a method that compensates for the energy losses at touchdown by virtual pre-compression of the SLIP model. These concepts are derived and presented 
in Section II, and applied to two different configurations of the model of an articulated leg developed at our lab (Fig. 1bc). In Section III, we show that the two systems are truly equivalent, apply the established angle of attack or stiffness dead-beat controllers to the model of a real robotic leg, and show that the bulk part of actuator torques can be produced by purely passive elastic elements.

\section{MethoDS}

\section{A. SLIP model}

A spring-loaded-inverted-pendulum (SLIP) is described by a point mass $m_{S L I P}$ that is attached to a mass-less prismatic spring with resting length $l_{0}$ and leg stiffness $k$ (Fig. 1(a)).

During flight phase, the model is only subject to the law of gravitation, and follows a parabolic trajectory. The leg length $l_{0}$ and angle of attack $\alpha_{0}$ are kept constant. When the leg strikes the ground, the motion of the $\mathrm{CoG}$ is redirected by the exerted spring force in the $\operatorname{leg} F_{\text {leg }}=k\left(l_{0}-l\right)$ that acts between a fixed ground contact point and the CoG:

$$
m \ddot{\mathbf{r}}_{S L I P}=\mathbf{F}_{\text {leg }}+m \mathbf{g} \text {, }
$$

where $\mathbf{r}_{S L I P}=[x, y]^{T}$ is the position of the point-mass and $\mathbf{g}=\left[0,-g_{E}\right]^{T}$ is the gravitational acceleration vector. While so far no analytical solution has been found for this differential equation, approximate solutions exist $[19,20]$.

\section{B. System Analysis: Apex return map}

The dynamics of the system are analyzed on a step-perstep basis, where one step is defined as the period between two successive apex transits ( $\dot{y}=0$ in flight). A motion is called periodically stable, if it is periodic (the system reaches the same apex height and forward velocity after one step) and if deviations from this periodic solution decrease from one step to the next. As the SLIP model is energetically conservative, apex height and forward speed are coupled by the given energy level. This reduces the stability problem to a single degree of freedom, which can be analyzed with the one-dimensional return map $y_{i+1}=P\left(y_{i}\right)$ that maps the height of two subsequent apex transits (single-step analysis). The stability of a fixed point $y_{i+1}=y_{i}=\mathrm{y}^{*}$ in this return map is determined by the slope of the map $\left.\frac{d y_{i+1}}{d y_{i}}\right|_{y_{i}=y^{*}}$ in this point. If it is smaller than one, an (energy conserving) error will vanish, and the solution is called stable. Any non-energy conserving error can be decomposed into a component that remains constant (as the system itself is not able to generate or destroy energy), and a component that is energy-conserving and hence follows the laws stated above. The region around a stable fixed point within which the system will return to the stable solution is called the basin of attraction.

\section{Articulated Leg}

The continuous mechanics of a robotic leg under support conditions can be described by

$$
\mathbf{M} \ddot{\mathbf{q}}+\mathbf{b}+\mathbf{g}+\mathbf{J}_{s}^{T} \mathbf{F}_{s}=\mathbf{S}^{T} \boldsymbol{\tau},
$$

with the mass matrix $\mathbf{M}$, the coriolis and centrifugal force vector $\mathbf{b}$, the gravitational force $\mathbf{g}$, the ground contact force $\mathbf{F}_{s}$ (with the corresponding support Jacobian $\mathbf{J}_{s}$ ), and the actuation torques $\boldsymbol{\tau}$ as well as the actuation matrix $\mathbf{S}$, which separates actuated (joints) from unactuated (main body position/orientation) generalized coordinates $\mathbf{q}$. For the simplest planar articulated leg with a fixed main body pitch, we have $\mathbf{q}=\left[\begin{array}{llll}x_{\text {MainBody }} & y_{\text {MainBody }} & \varphi_{\text {Hip }} & \varphi_{\text {Knee }}\end{array}\right]^{T}$ with an actuator selection matrix $\mathbf{S} \in \mathfrak{R}^{2 \times 4}$ that limits actuation to hip and knee joints $\left(\boldsymbol{\tau}=\left[\begin{array}{ll}\tau_{\text {hip }} & \tau_{\text {knee }}\end{array}\right]^{T}\right)$.

The contact force $\mathbf{F}_{s}$ is 0 during flight and can be eliminated during stance by introducing the contact condition

$$
\dot{\mathbf{x}}_{s}=\mathbf{J}_{s} \dot{\mathbf{q}}=0, \quad \ddot{\mathbf{x}}_{s}=\mathbf{J}_{s} \ddot{\mathbf{q}}+\dot{\mathbf{J}}_{s} \dot{\mathbf{q}}=0 .
$$

This leads to a support consistent description [21]

$$
\mathbf{M} \ddot{\mathbf{q}}+\mathbf{N}_{s}^{T}(\mathbf{b}+\mathbf{g})+\mathbf{J}_{s}^{T} \boldsymbol{\Lambda}_{s} \dot{\mathbf{J}} \dot{s} \dot{\mathbf{q}}=\left(\mathbf{S} \mathbf{N}_{s}\right)^{T} \boldsymbol{\tau},
$$

with the support space inertia matrix $\boldsymbol{\Lambda}_{s}=\left(\mathbf{J}_{s} \mathbf{M}^{-1} \mathbf{J}_{s}^{T}\right)^{-1}$ and the dynamically consistent support nullspace $\mathbf{N}_{s}=\left[\mathbf{I}-\mathbf{M}^{-1} \mathbf{J}_{s}^{T} \boldsymbol{\Lambda}_{s} \mathbf{J}_{s}\right]$. With this null-space, we can also express the instantaneous changes in velocities that occur when the leg strikes the ground at transition from flight to stance, and the contact point is brought to an immediate stop:

$$
\dot{\mathbf{q}}^{+}=\mathbf{N}_{s} \dot{\mathbf{q}}^{-}
$$

\section{Operational Space Control}

To impose the dynamics of a SLIP model onto such an articulated leg, we project the stance dynamics (including the support constraint) of the leg onto its aggregated center of gravity $\mathbf{r}_{C o G}$, according to Oussama Khatib's work on operational space control [18]:

$$
\mathbf{\Lambda}^{*} \ddot{\mathbf{r}}_{C o G}+\boldsymbol{\mu}^{*}+\mathbf{p}^{*}=\mathbf{F}
$$

When prescribing a desired CoG trajectory, we can use this formulation to compute the necessary operational space force $\mathbf{F}$, which is directly related to the required joint actuator torques $\tau$

$$
\boldsymbol{\tau}=\mathbf{J}^{* T} \mathbf{F}
$$

For a detailed derivation of the individual components of this projection, please refer to [21] and [22]. For completeness of this paper, a short definition of each element is presented in the following:

(i) support reduced Jacobian:

$$
\mathbf{J}^{*}=\mathbf{J}_{C o G} \mathbf{M}^{-1}\left(\mathbf{S} \mathbf{N}_{s}\right)^{T}\left(\mathbf{S} \mathbf{N}_{s} \mathbf{M}^{-1}\left(\mathbf{S} \mathbf{N}_{s}\right)^{T}\right)^{-1}
$$


(ii) task inertia:

$$
\boldsymbol{\Lambda}^{*}=\left(\mathbf{J M}^{-1} \mathbf{S} \mathbf{N}_{s} \mathbf{J}^{*}\right)^{-1}
$$

(iii) projected coriolis and centrifugal terms:

$$
\boldsymbol{\mu}^{*}=\boldsymbol{\Lambda}^{*} \mathbf{J}_{C o G} \mathbf{M}^{-1} \mathbf{N}_{s}^{T} \mathbf{b}-\boldsymbol{\Lambda}^{*} \dot{\mathbf{J}}_{C o G} \dot{\mathbf{q}}+\boldsymbol{\Lambda}^{*} \mathbf{J}_{C O G} \mathbf{M}^{-1} \mathbf{J}_{s}^{T} \boldsymbol{\Lambda}_{s} \dot{\mathbf{J}_{s}} \dot{\mathbf{q}}(10)
$$

(iv) projected gravitational part:

$$
\mathbf{p}^{*}=\boldsymbol{\Lambda}^{*} \mathbf{J}_{C G G} \mathbf{M}^{-1} \mathbf{N}_{s}^{T} \mathbf{g}
$$

\section{E. SLIP dynamics as a control law}

During ground contact, the dynamics described by the SLIP model can now be directly imposed to the CoG motion of the robotic leg. Solving (1) for $\ddot{\mathbf{r}}_{S L I P}$ and inserting the result into (6) and (7) leads to the SLIP motion control law for any arbitrary robotic leg:

$$
\boldsymbol{\tau}=\mathbf{J}^{* T}\left(\boldsymbol{\Lambda}^{*} \frac{1}{m}\left(\mathbf{F}_{l e g}+m \mathbf{g}\right)+\boldsymbol{\mu}^{*}+\mathbf{p}^{*}\right)
$$

Assuming that we have a perfect model and a feasible $\mathrm{CoG}$ motion described by the SLIP model, the $\mathrm{CoG}$ of the articulated leg will perfectly follow this prescribed motion. The first part of the sum in (12) assures thereby the correct CoG motion under support constraint, while $\boldsymbol{\mu}^{*}$ and $\mathbf{p}^{*}$ compensate for gravitational and dynamic coupling effects that are due to the articulated design.

During flight phase, the ballistic CoG curve is inherently ensured by the lack of ground contact forces (constant impulse in $\mathrm{x}$-direction, gravity influence in y direction). The correct leg length and angle of attack for touchdown are easily achieved using inverse kinematic position control at joint level.

\section{F. Kinematic Rectification}

In contrast to the traditional SLIP model (spring between CoG and foot point), one could argue to use a kinematic equivalent SLIP model with a linear torsion spring in the knee joint [17]. This results in a linear stiffness as a function of the actual spring length $l$, segment length $s_{\text {seg }}$, and rotational stiffness $c_{\text {rot }}$ :

$$
\begin{aligned}
& \varphi_{\text {knеe }}(l)=2 \cdot \arccos \left(\frac{l}{2 s_{\text {seg }}}\right) \\
& k(l)=\frac{c_{\text {rot }}}{s_{\text {seg }}} \cdot \frac{\varphi_{\text {knee }}\left(l_{0}\right)-\varphi_{\text {knee }}(l)}{\left(l-l_{0}\right) \sin \left(\varphi_{\text {knee }}(l) / 2\right)}
\end{aligned}
$$

We will defer this idea until the final conclusion.

\section{G. Impact at landing}

In contrast to a SLIP model with a mass-less prismatic spring, a real robotic leg suffers from impacts at landing. Normal and tangential impulse substantially change the velocity at impact according to (5). The post-impact CoG velocity can be expressed as a function of the SLIP parameters $\left(\alpha, l_{0}\right)$ and the pre-impact velocity $\dot{\mathbf{r}}_{C O G}^{-}$

$$
\dot{\mathbf{r}}_{C o G}^{+}=\underbrace{\mathbf{J}_{C o G} \mathbf{N}_{S} \mathbf{J}_{C O G}^{\#}}_{\mathbf{C}\left(\alpha, l_{0}\right)} \dot{\mathbf{r}}_{C o G}^{-}
$$

with $J_{C o G}^{\#}$ being the inverse of the CoG Jacobian under the condition of zero pre-impact joint velocity. Unfortunately, this velocity change entails also an energy loss. A comparison of different robotic legs with known mass and geometric parameters (Stanford Kolt [23-25], OLIE [26, 27], the leg of Curran and Knox $[28,29]$ and the two legs developed at our Lab $[30,31])$ at a reasonable inner knee elongation angle of about $120^{\circ}\left(180^{\circ}\right.$ would be completely stretched) shows an energy loss of $12 \pm 4 \%$, which is drastically increasing the more we extend the knee joint. While the rather theoretical SLIP model achieves continuous hopping without actuation, energy has to be introduced in the non-mass-less case to compensate for these losses. There exist many strategies from an optimal control point of view (e.g. minimization of positive actuator work) to achieve this, but they would all cause the system to deviate from the desired SLIP dynamics.

To circumvent this problem, we propose the use of a modified SLIP model with impact compensation (SLIPic), which differs from the regular SLIP model only in the fact that the leg spring is being pre-compressed at touch-down. I.e. at the instance of landing, the resting length of the leg spring is changed to $l_{0}^{+}=l_{0}^{-}+\Delta l$, which increases the system energy according to

$$
\Delta E=0.5 k \Delta l^{2}
$$

This energy matches the loss of kinetic energy of the CoG point in the collision, which must equal:

$$
\begin{aligned}
\Delta E & =0.5 m_{C O G}\left(\left|\dot{\mathbf{r}}_{C O G}^{-}\right|^{2}-\left|\dot{\mathbf{r}}_{C O G}^{+}\right|^{2}\right) \\
& =0.5 m_{C O G} \dot{\mathbf{q}}^{-T}\left(\mathbf{J}_{C O G}^{T} \mathbf{J}_{C O G}-\mathbf{N}_{s}^{T} \mathbf{J}_{C O G}^{T} \mathbf{J}_{C O G} \mathbf{N}_{s}\right) \dot{\mathbf{q}}^{-}
\end{aligned}
$$

In other words, instead of tracking the dynamics of a single SLIP model, we first track the dynamics of a model with leg length $l_{0}^{-}$(which defines the kinematic positions at touchdown) and then switch to a model with leg length $l_{0}^{-}+\Delta l$ (which defines the ground contact forces during stance and the leg length at lift-off). The difference $\Delta l$ compensates for the energetic losses that happen at the same instance. I.e., SLIPic is energetically conservative, even in the presence of impact losses. The detailed view of Fig. 4a depicts the instantaneous transfer of the lost kinetic energy into the virtual spring energy at the point of collision.

\section{H. Dead beat high level control and SLIPic model}

There exist various control strategies for continuous height and speed running with SLIP models in different terrain $[14,15]$, and all of them can be directly applied to SLIPic. The general idea is to solve the mapping

$$
[y, \dot{x}]_{i} \stackrel{\text { SLIPdynamics }}{\longrightarrow}[y, \dot{x}]_{i+1}
$$

as a function of the angle of attack $\alpha$ and the spring stiffness $k$. Depending on the desired change in apex height and velocity (which must be energetically conservative), $\alpha$ or $k$ are adapted to reach the goal state (index $\mathrm{i}+1$ ) 


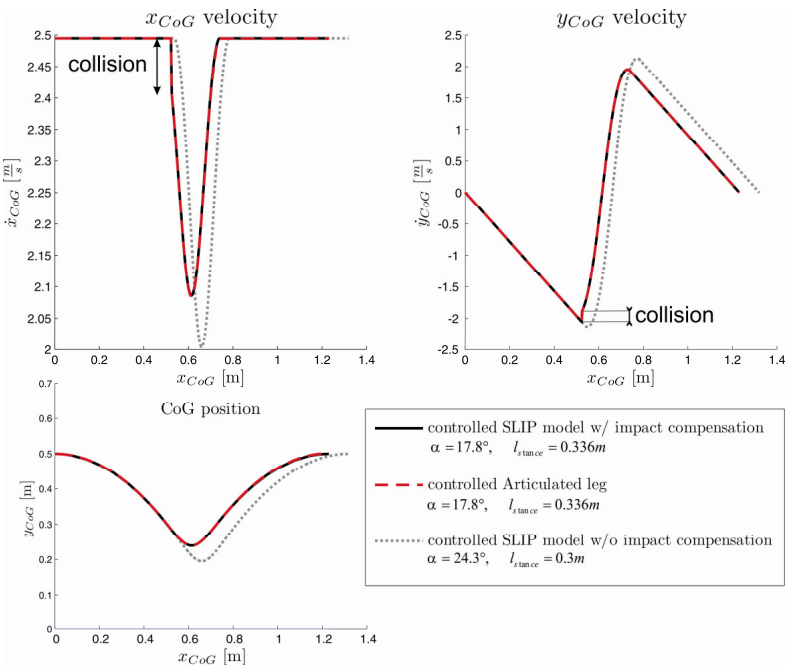

Fig. 2. The articulated leg (red dashed) and its equivalent SLIPic model (black solid) running with the same parameters $\alpha=17.8^{\circ}$ and $l=0.336 m$ perform exactly the same CoG motion. As a comparison, a SLIP model without impact compensation (grey dotted) is controlled at the same fix point with the parameters $\alpha=24.3^{\circ}$ and $l=0.3 \mathrm{~m}$. While having the same flight curve, there is no instantaneous velocity change due to the impact at landing

within one single hop (dead beat). Since the stance phase dynamics are not solvable in closed form, this must be done numerically by repeated simulation or with an approximate analytical solution $[19,20]$. However, as the SLIP dynamics are fairly simple this is a computationally feasible task.

With the presented framework, the exact same control approach can now be applied to an actual robotic leg, by computing (18) for the SLIPic model, and imposing the resulting dynamics on the actual leg. Except for the two additional equations (15) and (17), solving this control problem is computationally as expensive as solving the map (18) for a SLIP model and hence possible in real time.

\section{RESULTS}

\section{A. Model equivalence}

In a first set of trials, we compared the motion of a SLIPic model with the simulation of an articulated robotic leg to which we applied the proposed operational space controller. Fig. 2 shows that the SLIPic model (black solid line) behaves as expected and is exactly followed by the low level controlled articulated leg (red dashed line). Both models were started with the same initial conditions of the $\mathrm{CoG}$ and same model parameters mass $m$, angle of attack $\alpha$, spring constant $k$, and spring length $l_{0}$.

For comparison, a SLIP model without impact compensation (grey dotted line) was simulated in parallel. While the ballistic flight curve is of course identical, a different angle of attack $\left(24.3^{\circ}\right.$ compared to $17.8^{\circ}$ for the articulated leg) had to be chosen, such that the apex height remains constant for continuous hopping.
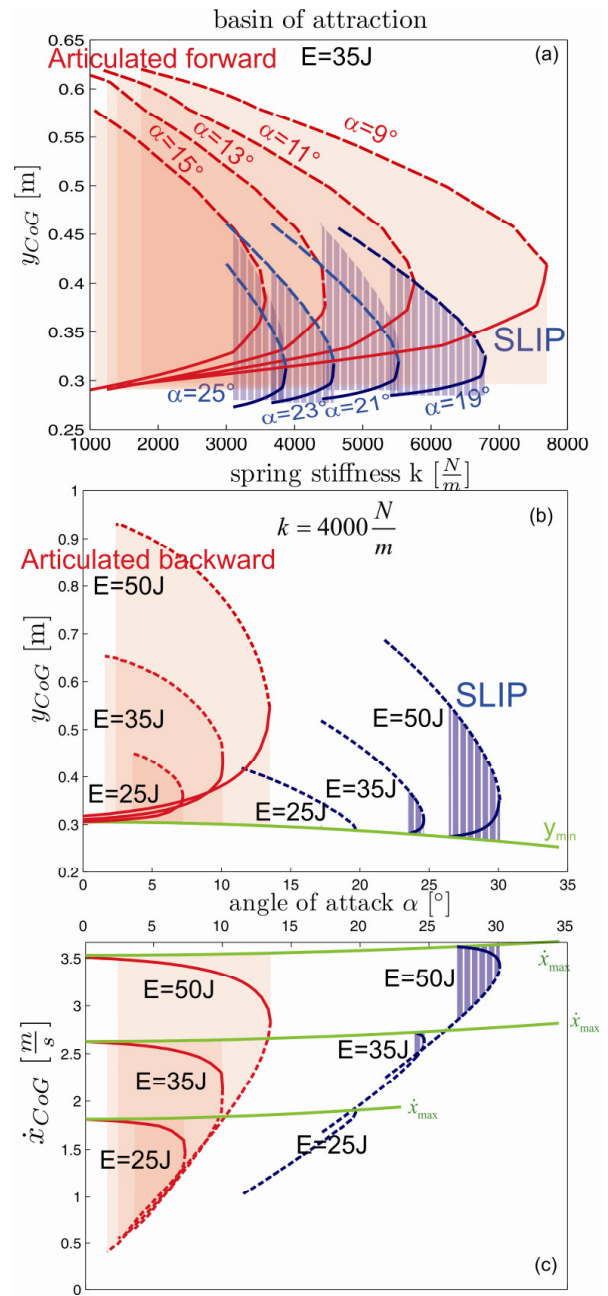

Fig. 3. The basin of attraction for the SLIPic and the SLIP model were calculated as a function of the angle of attack $\alpha$, spring stiffness $k$ and energy level E. The SLIPic equivalent of an articulated leg in forward as well as backward configuration shows a much larger basin of attraction than the SLIP model due to the collision at landing. Stable fixed points are found at lower speeds.

\section{B. Self-stabilization}

Various studies have shown the existence of self-stabilizing effects in the SLIP model. For a certain combination of stiffness, angle of attack, and energy level, stable fixed points, respectively basin of attraction can be found. Similar studies have been performed with segmented legs, whereby segment mass and inertia effects (and consequently collision effects) were completely neglected. We performed the same investigations with the SLIPic-equivalent of a real articulated robotic leg. Stable and instable fixed points (that define the basin of attraction) were found by varying the angle of attack $\alpha$, spring stiffness $k$, energy level $E$ and initial apex height $y_{i}$.

In Fig. 3a, the basin of attraction of a SLIP runner and an articulated leg in forward motion (Fig. 1c) are compared. The articulated leg (respectively its SLIPic equivalent) shows stable fixed points for a larger variety of parameters resulting in a substantial increase of the basin of attraction. 
Similarly, when keeping the stiffness constant and identifying the self-stabilizing area for different energy levels, the real robotic leg shows stable fixed points for a larger parameter and initial condition set. Stable operation with low energy and low forward speed is only possible with the articulated leg.

\section{Dead beat controlled hopping}

Continuous hopping on uneven terrain requires a step-tostep adaptation of the system parameters $\alpha$ or $k$ depending on the ground level height. Established strategies [14, 15] relay on searching fixed points in the apex height return map. We implemented an impact angle control for the articulated robotic leg. The stiffness was downscaled from the stiffness found in human running [11] to our robotic leg size. The fix point search as a function of the impact angle and energy level is done using the SLIPic model and holds as the input for the low level operational space controller of the real robotic leg. Fig. 4 shows the expected dead beat behavior of the articulated leg for hopping on uneven terrain. During ground contact, energy is stored in the virtual spring and apex height as well as forward velocity is kept constant. In addition to the purely passive SLIP model, we can change the energy level of the articulated leg. This is done in the same natural way of an additional spring pre-compression at the point of impact.

\section{CONCLUSION}

In this paper we extended the established SLIP model to include systems which are not energetically conservative but are subject to impact losses at touchdown and introduced the impact compensating SLIPic-model. We then used a low level operational space controller to impose the SLIPic dynamics onto an actual robotic leg. In combination, these two steps allow the application of well established high level dead beat controllers to real robots.

The main motivation of using SLIP models to control legged robots originates from the simple but still very accurate description of human and animal biomechanics. Exhaustive research based on human running data has shown that SLIP dynamics is more than just a metaphor; they literally describe the $\mathrm{CoG}$ control target. In other words, SLIP models are a good description of the natural dynamics of highly complex systems. Furthermore, theoretical research in the biomechanics field yielded advantageous properties such as dynamic stability and the possibility for dead beat control.

Using the presented approach of this paper is a substantial step towards closing the gap between these theoretical foundations and the application of SLIP controllers in real robots. In contrast to [32] were the virtual leg behavior must be as close as possible to the SLIP dynamics, we ensure SLIP equal motion of the CoG for arbitrary robotic legs using a low level operational space controller. The whole articulated leg dynamics can now be described by a SLIP model including impact compensation. This has the great
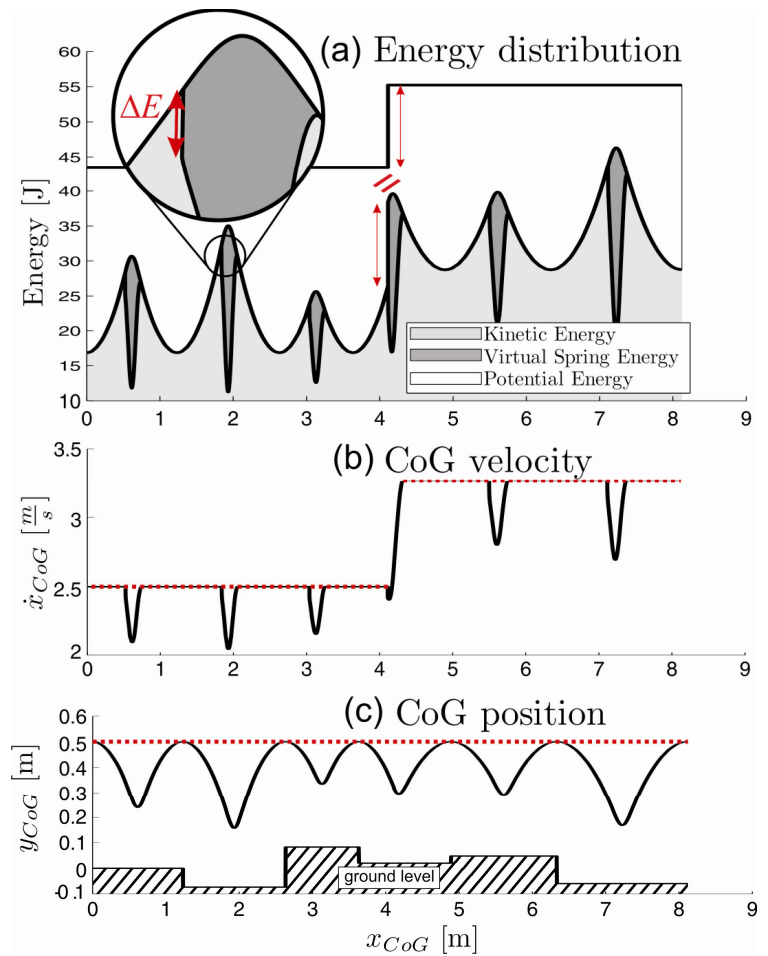

Fig. 4. Continuous hopping on uneven ground is achieved with a deadbeat controller that adjusts the angle of attack. The spring is precompressed with the amount of energy that is lost in collision. (a) shows the energy distribution between kinetic, potential, and virtual spring energy. At the fourth stance period, the energy level is changed by adding an additional amount of spring pre-compression resulting later in an increase of kinetic energy. (b) Consequently, a new forward speed is achieved while (c) the apex height is kept constant for a varying ground level.

advantage that all established SLIP dead beat controllers can be applied with nearly the same computation effort for the theoretical SLIPic model as well as for real robotic legs.

Furthermore, studies of the disturbance dynamics of an articulated leg under the influence of collisions were performed. A large increase in stable fixed points and basins of attractions compared to the SLIP model were found. These results are similar to those documented in [17], but must have a different offspring. In [17], the use of mass-less legs, limits the explanation to the nonlinear relation between the force acting on the point mass and the torque in the knee. The results in this study with a real robotic leg are different. We have a SLIP equal stance phase with a linear stiffness. The stabilization effects must hence originate entirely from the collision. A somehow intuitive explanation is that an increase in forward velocity automatically results in a higher impact in the same direction and consequently in larger $\mathrm{CoG}$ speed change in that direction (which equivalently holds for the normal direction).

So far, we are only controlling the joint torques and completely neglect the actuator side. We based the mechanical parameters on our recently developed robotic leg driven by series elastic actuators. Considering passive dynamics and hence energy efficiency, most of the joint torque should be provided by the torsion joint spring itself. 
a) linear SLIPic mode

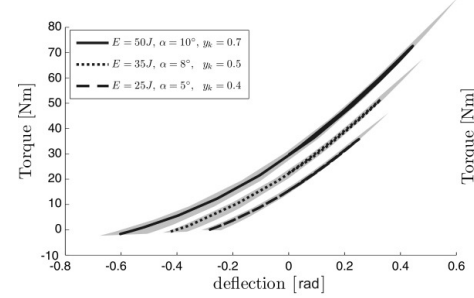

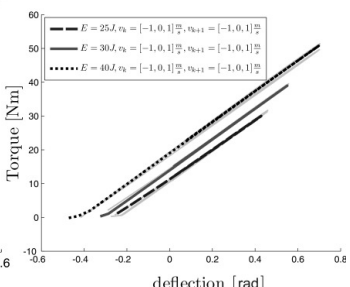

b) rectified SLIPic model

Fig. 5. The torque-deflection curve at the knee joint is plotted for hopping at three different energy levels. As these curves show virtually no hysteresis, the necessary torques can be generated passively, for example by an appropriately tuned spring. The kinematic rectification motivated in Section II.F shows that despite all dynamic effects, linear springs in the joint can be utilized (b). The spring characteristics are only depending on the absolute energy level and are nearly indifferent to changes in the forward velocity and jumping height. The shaded area under each curve illustrates the range of characteristics that are necessary when both parameters are changed.

Fig. 5 shows the torque-angle relationship that is necessary on joint level for hopping on different energy levels. As these curves show virtually no hysteresis, the necessary torques could be generated to a large part passively, thus reducing the necessary actuator power and energy consumption.

Additionally, the necessary characteristics only depend on the selected energy level and are nearly independent from hopping height and velocity. Which further facilitates the use of passive elements and thus paves the road for highly efficient legged locomotion

\section{REFERENCES}

[1] R. M. Alexander, "3 Uses for Springs in Legged Locomotion," International Journal of Robotics Research, vol. 9, pp. 53-61, April 1990.

[2] R. Blickhan, "The spring-mass model for running and hopping," Journal of Biomechanics, vol. 22, pp. 1217-1227, 1989.

[3] R. Blickhan and R. J. Full, "Similarity in multilegged locomotion: Bouncing like a monopode," Journal of Comparative Physiology A: Neuroethology, Sensory, Neural, and Behavioral Physiology, vol. 173, pp. 509-517, 1993.

[4] R. Full and D. Koditschek, "Templates and anchors: neuromechanical hypotheses of legged locomotion on land," $J$ Exp Biol, vol. 202, pp. 3325-3332, December 1, 19991999.

[5] G. A. Cavagna, N. C. Heglund, and C. R. Taylor, "Mechanical work in terrestrial locomotion: two basic mechanisms for minimizing energy expenditure," AJP Regul Integr Comp Physiol, vol. 233, pp. 243-261, November 11977.

[6] M. H. Dickinson, C. T. Farley, R. J. Full, M. A. R. Koehl, R. Kram, and S. Lehman, "How Animals Move: An Integrative View," Science, vol. 288, pp. 100-106, April 72000.

[7] M. H. Raibert, Legged robots that balance. Cambridge, Mass.: MIT Press, 1986.

[8] J. Schmitt and P. Holmes, "Mechanical models for insect locomotion: dynamics and stability in the horizontal plane I. Theory," Biological Cybernetics, vol. 83, pp. 501-515, 2000.

[9] R. M. Ghigliazza, R. Altendorfer, P. Holmes, and D. Koditschek, "A simply stabilized running model," Siam Review, vol. 47, pp. 519549, Sep 2005

[10] A. Seyfarth, H. Geyer, M. Gunther, and R. Blickhan, "A movement criterion for running," Journal of Biomechanics, vol. 35, pp. 649655, May 2002.

[11] H. Geyer, R. Blickhan, and A. Seyfarth, "Natural dynamics of spring-like running: emergence of selfstability," in (CLAWAR), London, UK, 2002.
[12] H. Geyer, A. Seyfarth, and R. Blickhan, "Compliant leg behaviour explains basic dynamics of walking and running," Proceedings of the Royal Society B: Biological Sciences, vol. 273, pp. 2861-2867, 2006.

[13] J. E. Seipel and P. Holmes, "Running in Three Dimensions: Analysis of a Point-mass Sprung-leg Model," The International Journal of Robotics Research, vol. 24, pp. 657-674, August 1, 20052005.

[14] A. Seyfarth and H. Geyer, "Natural control of spring-like running optimized self-stabilization," in International Conference on Climbing and Walking Robots (CLAWAR 2002), London, 2002, pp. 81-85.

[15] M. Ernst, H. Geyer, and R. Blickhan, "Spring-Legged Locomotion on Uneven Ground: A Control Approach to Keep the Running Speed Constant," in International Conference on Climbing and Walking Robots (CLAWAR), Istanbul, Turkey, 2009, pp. 639-644.

[16] A. Seyfarth, M. Günther, and R. Blickhan, "Stable operation of an elastic three-segment leg," Biological Cybernetics, vol. 84, pp. 365382, 2001.

[17] J. Rummel and A. Seyfarth, "Stable Running with Segmented Legs," The International Journal of Robotics Research, vol. 27, pp. 919934, August 1, 20082008.

[18] O. Khatib, "A unified approach for motion and force control of robot manipulators: The operational space formulation," Robotics and Automation, IEEE Journal of, vol. 3, pp. 43-53, 1987.

[19] H. Geyer, A. Seyfarth, and R. Blickhan, "Spring-mass running: simple approximate solution and application to gait stability," Journal of Theoretical Biology, vol. 232, pp. 315-328, 2005.

[20] W. J. Schwind and D. E. Koditschek, "Approximating the Stance Map of a 2-DOF Monoped Runner," Journal of Nonlinear Science, vol. 10, pp. 533-568, 2000.

[21] L. Sentis, "Compliant Control of Whole-Body Multi-Contact Behaviors in Humanoid Robots," in Motion Planning for Humanoid Robots: Springer Global Editorial, 2009.

[22] L. Sentis, "Synthesis and Control of Whole-Body Behaviors in Humanoid Systems," Stanford University, 2007.

[23] J. G. Nichol, S. P. N. Singh, K. J. Waldron, L. R. Palmer, III, and D. E. Orin, "System Design of a Quadrupedal Galloping Machine." vol. 23, 2004, pp. 1013-1027.

[24] J. G. Nichol, "Design for Energy Loss and Energy Control in a Galloping Artificial Quadruped," in Mechanical Engineering. vol. PhD Stanford: Stanford University, 2005, p. 159.

[25] J. G. Nichol and K. J. Waldron, "Biomimetic Leg Design for Untethered Quadruped Gallop," 2006.

[26] H. De Man, D. Lefeber, and J. Vermeulen, "Hopping on Irregular Terrain with One Articulated Leg," in International Symposium on Robotics (ISR) Birmingham, United Kingdom, 1998.

[27] H. De Man, D. Lefeber, and J. Vermeulen, "Design and Control of a Robot with One Articulated Leg for Locomotion on Irregular Terrain," in CSIM courses and lectures. vol. 405, A. Morecki, G. Bianchi, and M. Wojtyra, Eds.: SpringerWienNewYork, 1998, pp. 417-424.

[28] B. T. Knox, "Evaluation of a Prototype Series-Complient Hopping Leg for Biped Robot Applications," in Department of Mechanical Engineering: The Ohio State University, 2007, p. 127.

[29] S. Curran and D. E. Orin, "Evolution of a jump in an articulated leg with series-elastic actuation," in IEEE International Conference on Robotics and Automation (ICRA) Pasadena, CA, USA, 2008, pp. 252-258.

[30] M. Hutter, C. D. Remy, and R. Siegwart, "Design of an Articulated Robotic Leg with Nonlinear Series Elastic Actuation," in $12^{\text {th }}$ International Conference on Climbing and Walking Robots (CLAWAR), Istanbul, Turkey, 2009.

[31] M. Hutter, C. D. Remy, and R. Siegwart, "Adaptive Control Strategies for Open-loop Dynamic Hopping," in IEEE/RSJ International Conference on Intelligent RObots and Systems (IROS), St. Louis, MO, USA, 2009.

[32] U. Saranli, W. J. Schwind, and D. E. Koditschek, "Toward the control of a multi-jointed, monoped runner," in Robotics and Automation, 1998. Proceedings. 1998 IEEE International Conference on, 1998, pp. 2676-2682 vol.3. 